\title{
Single-Molecule Transport of Fullerene-based
}

\section{Curcuminoids}

Diana Dulić, ${ }^{a, *}$ Alfredo Rates, ${ }^{a, c}$ Edison Castro, ${ }^{b}$ Jacqueline Labra-Muñoz, ${ }^{a}$ Daniel Aravena, ${ }^{d}$ Alvaro Etcheverry-Berrios, ${ }^{e}$ Daniel Riba-López, ${ }^{f}$ Eliseo Ruiz,g Núria Aliaga-Alcalde, ${ }^{f, h}$ Monica Soler, ${ }^{e}$ Luis Echegoyen, ${ }^{b}$ and Herre S. J. van der Zant, ${ }^{c}$

a) Department of Physics and Department of Electrical Engineering, Faculty of Physical and Mathematical Sciences, University of Chile, Avenida Blanco Encalada 2008, Santiago 8330015, Chile.

b) Department of Chemistry, University of Texas, 500 West University Avenue, El Paso, Texas 79968, United States.

c) Kavli Institute of Nanoscience, Delft University of Technology, Lorentzweg 1, Delft 2628 CJ, The Netherlands.

d) Department of Material Chemistry, Faculty of Chemistry and Biology, University of Santiago of Chile, Casilla 40, Correo 33, Santiago 9170022, Chile.

e) Department of Chemical Engineering, Biotechnology and Materials, Faculty of Physical and Mathematical Sciences, University of Chile, Beauchef 851, Santiago, 837.0415, Chile.

f) Institut de Ciència de Materials de Barcelona (CSIC-ICMAB), Campus de la Universitat Autònoma de Barcelona, 08193 Bellaterra, Spain.

g) Departament de Química Inorgànica i Orgànica and Institut de Recerca de Química Teòrica i Computacional, Universitat de Barcelona, Diagonal 645, 08028 Barcelona, Spain.

h) ICREA (Institució Catalana de Recerca i Estudis Avançats), Passeig Lluís Companys 23, 08010 Barcelona, Spain. 


\begin{abstract}
We present experimental and theoretical studies of the single-molecule conductance through non-planar fullerocurcuminoid molecular dyads in ambient using the mechanically controllable break junction technique. We show that molecular dyads with bare fullerenes form configurations with conductance features related to different transport channels within the molecules, as identified with filtering and clustering methods. The primary channel corresponds to charge transport through the methylthio terminated backbone. Additional low-conductance channels involve one backbone side and the fullerene. In fullerenes with attached four equatorial diethyl malonate groups the latter transport pathway is blocked. Density Functional Theory calculations corroborate the experimental observations. In combination with NonEquilibrium Green Functions the conductance values of the fullerocurcuminoid backbones are found to be similar to those of a planar curcuminoid molecule without a fullerene attached. In the non-planar fullerocurcuminoid systems the highestconductance peak occurs partly through space, compensating for the charge delocalization loss present in the curcuminoid system.
\end{abstract}

KEYWORDS: molecular electronics, single-molecule conductance, quantum charge transport, curcuminoids, fullerenes, quantum chemistry calculations.

TOC GRAPHICS 


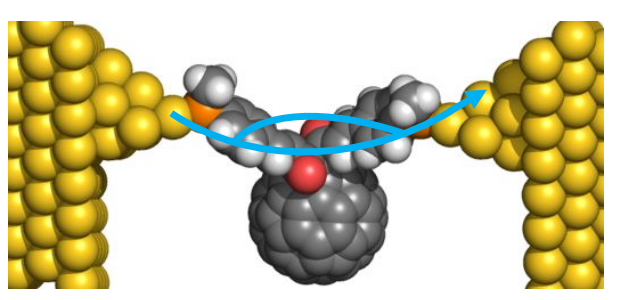

Due to their unique electronic properties, $\mathrm{C}_{60}$ and its derivatives represent ideal candidates for molecular-based devices. ${ }^{1,2}$ Thus far, the synthesis of a wide variety of fullerene derivatives have been reported and their potential applications as electronic, magnetic, catalytic, biological and optical materials have been investigated. ${ }^{3-9}$ At the single-molecule level, establishing robust electronic functionalities in fullerene derivatives connected to gold electrodes have, however, proven to be a challenging task. Pristine $\mathrm{C}_{60}$ has been studied using both scanning tunneling microscope breakjunctions ${ }^{10}$ and mechanically controllable break junction (MCBJ) techniques. ${ }^{11,12,13}$ Conductance values around $0.1 \mathrm{G}_{0}$ have been reported, where $\mathrm{G}_{0}$ is the quantum of conductance equaling $2 e^{2} / h=77.48 \mu \mathrm{S}$, where $e$ is the elementary charge and $h$ is Planck's constant, in agreement with theoretical calculations. ${ }^{14,15}$

Taking advantage of its affinity to metals, various groups have studied 'dumbbell' benzene-difullerene derivatives in which $\mathrm{C}_{60}$ 's are placed at both ends of the molecule and act as the anchoring groups to the metal leads. ${ }^{16,17,18} \mathrm{~A}$ conductance value around $10^{-4} \mathrm{G}_{0}$ was reported in these cases. The lower conductance value compared to that of pristine $\mathrm{C}_{60}$ was attributed to a charge transport limiting barrier created by the nitrogen atoms of the pyrrolidine bridging the benzene backbone to the $\mathrm{C}_{60}$ end groups. A more recent study of a dumbbell fullerene derivative shows two different electronic transport configurations, one assigned to transport through the molecular 
bridge and the second, at higher conductance values, ascribed to a single $\mathrm{C}_{60}$ anchoring group trapped between the two adjacent electrodes. ${ }^{19}$ Additional experimental studies of amino ${ }^{20}$ and diazofluorene ${ }^{21} \mathrm{C}_{60}$ terminated derivatives also show multiple conductance configurations.

Here, we present experimental and theoretical charge transport studies of the singlemolecule conductance through fullerocurcuminoid $\left(\mathrm{CCM}-\mathrm{C}_{70} / \mathrm{C}_{60}\right)$ molecular dyads (see Fig. 1). Me-S terminated CCM's were chosen because they form stable and well-defined molecular junctions. ${ }^{22,23}$ Three systems with the CCM skeleton connected to a $\mathrm{C}_{70}, \mathrm{C}_{60}$ and to a $\mathrm{C}_{60}$ fullerene all-equatorial tetramalonate derivative $\left(\mathrm{CCM}-\mathrm{C}_{70}, \mathrm{CCM}-\mathrm{C}_{60}\right.$ and $\mathrm{CCM}-\mathrm{C}_{60} \mathrm{P}$, respectively) have been investigated. In the latter, four equatorial diethyl malonate groups were added to the $\mathrm{C}_{60}$. The ethyl esters in these groups are expected to have a high contact resistance and low affinity for gold, thus modifying the interaction between the fullerene moiety and the electrodes in comparison with the non-equatorially modified molecule $\left(\mathrm{CCM}-\mathrm{C}_{60}\right)$. We find that the presence of the fullerenes attached to the CCM backbone lowers the conductance only slightly with respect to the single CCM's without fullerenes attached to them. A priori the results were unexpected, due to the dramatic conformational changes that occur to the curcuminoid backbone in $C C M-C_{70}, C C M-C_{60}$ and $C C M-C_{60} P$, where the $C C M$ structure changes from almost planar to one with a distinct V-shape in the fullerocurcuminoid systems, with a corresponding change in charge transfer pathway (see Fig. 1). In addition, the two unprotected fullerene-based derivatives show multiple junction configurations at lower conductance values, attributed to junction formation between one of the Me-S groups and the respective fullerene moiety; this second charge transport pathway is not found for $C \mathrm{CM}-\mathrm{C}_{60} \mathrm{P}$, showing that the diethyl malonate groups effectively disrupt the direct interaction of the fullerene to the gold electrodes. 

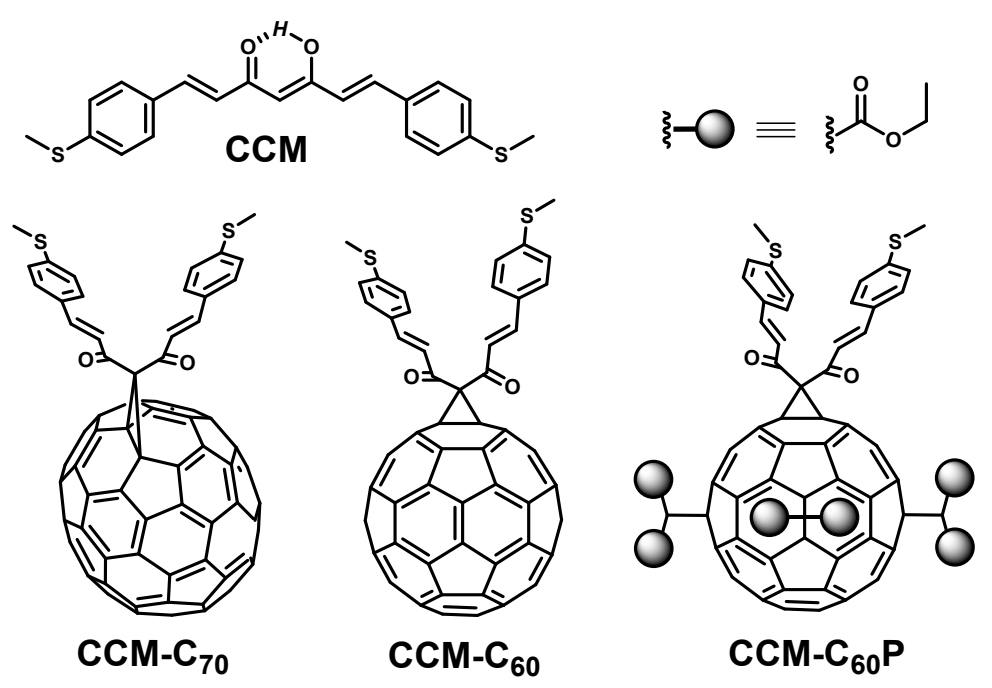

Figure 1. Chemical structures of the curcuminoid (CCM) derivatives used in this study.

$\mathrm{CCM}-\mathrm{C}_{70}$ and $\mathrm{CCM}-\mathrm{C}_{60}$ were synthesized following a reported procedure. ${ }^{24} \mathrm{CCM}-\mathrm{C}_{60} \mathrm{P}$ was synthesized in a multistep topologically controlled approach, ${ }^{25}$ see supporting information for the detailed synthesis. The conductance of the CCM-fullerenes (Fig. 2) was measured in air at room temperature using the $\mathrm{MCBJ}$ technique explained in more detail elsewhere. ${ }^{26,27}$ Briefly, a narrow gold constriction is fabricated by e-beam lithography on a flexible substrate, underetched and subsequently mounted in a setup equipped with a three-point bending mechanism. Bending the substrate causes the suspended gold wire to stretch until a single gold atom connects two thicker parts of the gold wire. Further bending results in a breaking of the wire thereby forming two sharp electrodes. The single-gold atom termination can be observed in a conductance vs. electrode displacement trace (breaking trace) as a short plateau around $1 \mathrm{G}_{0}$. $\mathrm{A}$ sudden drop in conductance to about $10^{-3} \mathrm{G}_{0}$ signals the rupture of the gold wire. This point is defined as the zero displacement in a breaking trace. After the initial opening of the junction, the electrodes are moved further apart, and the conductance is recorded until the measured value reaches the noise level. When this sequence has been finished, the electrodes are fused forming a continuous wire again with a conductance of more than $20 \mathrm{G}_{0}$ and the whole process is repeated in an automated way. 
Prior to the deposition of the molecule of interest, each experiment starts by characterizing the bare device. A bias voltage of $0.1 \mathrm{~V}$ is applied across the gold wire and the current passing through it is measured while repeatedly opening and closing the junction. Devices are used for molecule measurements only if they show just vacuum tunneling behavior and a clear sign of single-gold atom contact, i.e., a clear 1 $\mathrm{G}_{0}$ plateau. A $30 \mu \mathrm{M}$ solution with the molecule under investigation is then prepared by dissolving the starting compound in dichloromethane. Two $2 \mu \mathrm{L}$ droplets of the solution are drop-casted and dried on the freshly characterized device; subsequently, thousands of consecutive breaking traces are measured in an automated way.

To facilitate the identification of molecular traces, we applied two types of statistical analyses: a filtering method (method I), based on conductance plateau recognition, and an unsupervised clustering ${ }^{28}$ method (method II), both described in the SI. Figure 2 displays filtered (method I) two- (Fig. 2a,c,e) and one- (Fig. 2b,d,f) dimensional conductance histograms for $\mathrm{CCM}-\mathrm{C}_{70}, \mathrm{CCM}-\mathrm{C}_{60}$ and $\mathrm{CCM}-\mathrm{C}_{60} \mathrm{P}$, respectively. Datasets are composed of breaking traces from individual junctions recorded with the same settings. Insets of Fig. 2a,c,e represent 2D histograms constructed from the traces that were excluded in the filtering procedure displaying the empty junctions. The sum of the two histograms, thus, constitutes the complete data sets, which can be found in Fig. S7. 
(a)

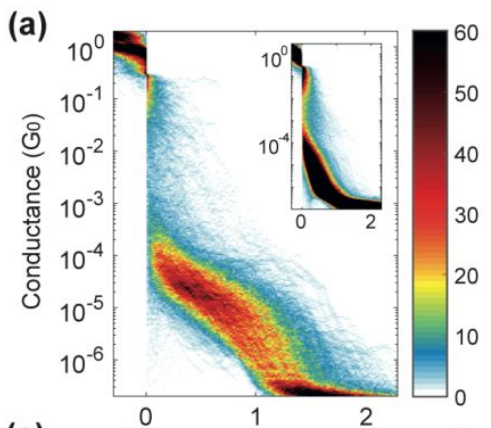

(c)
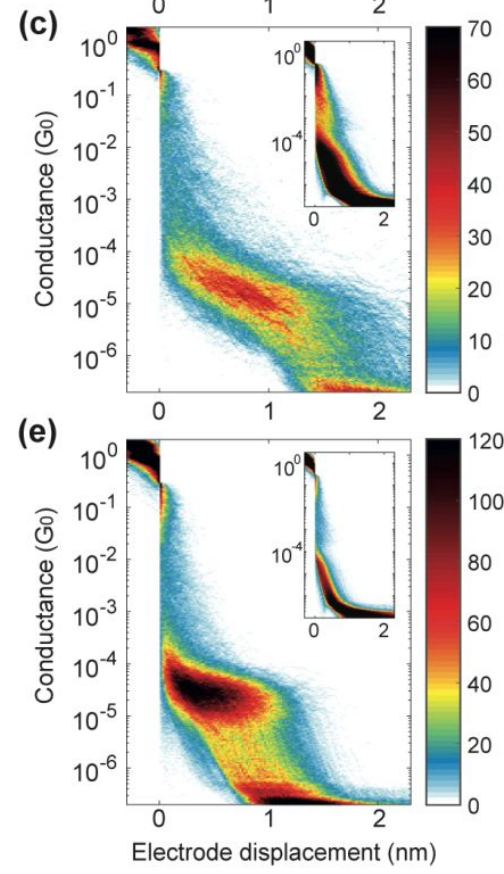

(b)

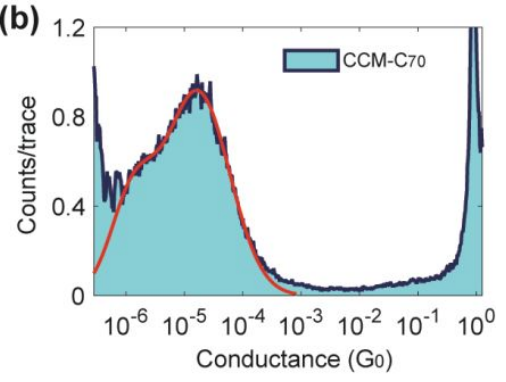

(d)

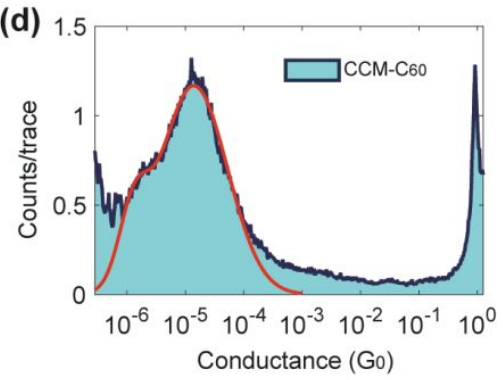

(f)

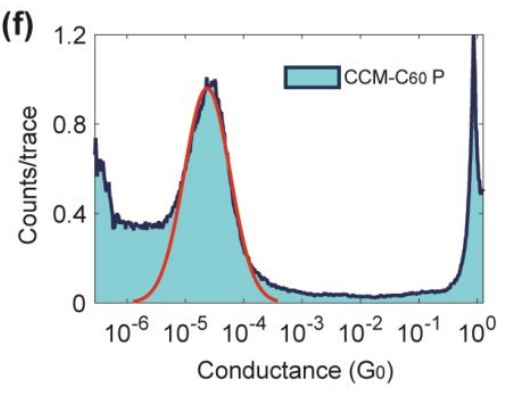

Figure 2. a), c), e). Two-dimensional conductance histograms built from filtration of 10.000 consecutive breaking traces recorded after drop-casting a solution containing $\mathrm{CCM}-\mathrm{C}_{70}, \mathrm{CCM}-\mathrm{C}_{60}$ and $\mathrm{CCM}-\mathrm{C}_{60} \mathrm{P}$, respectively. For the selection of the traces, method I was used. The insets show all the traces that were excluded in the filtering procedure. The applied bias voltage was $100 \mathrm{mV}$ and the measurement speed was 6.0 $\mathrm{nm} / \mathrm{s} . \mathbf{b}), \mathbf{d}), \mathbf{f})$. Normalized one-dimensional histograms obtained by integrating the breaking traces along the displacement axis of the filtered data in the corresponding 2D histograms (light blue areas) of $\mathrm{CCM}-\mathrm{C}_{70}, \mathrm{CCM}-\mathrm{C}_{60}$ and $\mathrm{CCM}-\mathrm{C}_{60} \mathrm{P}$, in that order. The red lines correspond to log-normal fits, which define the most probable conductance values listed in Table S1. 
The 2D histograms in Fig. 2a,c,e show high-counts regions between $10^{-4}$ and $10^{-5} \mathrm{G}_{0}$, which extend up to lengths of 1-1.5 nm. For CCM-C $\mathrm{C}_{70}$, the counts are mostly concentrated around two values and the log-normal fit to its one-dimensional histogram (Fig. 2b) indicates that the corresponding most probable conductance values (using method I, see Table S1) are $1.7 \times 10^{-5}$ and $1.3 \times 10^{-6} \mathrm{G}_{0}$. Log-normal fits of the one-dimensional histogram of $\mathrm{CCM}^{-\mathrm{C}_{60}}$ (Fig. 2d) result in two similar values for the most probable conductances: $1.4 \times 10^{-5}$ and $1.3 \times 10^{-6} \mathrm{G}_{0}$. In contrast, for CCM-C $60 \mathrm{P}$, the 1D histogram of Fig. $2 \mathrm{f}$ only shows one clear peak, with a conductance value of $2.4 \mathrm{x}$ $10^{-5} \mathrm{G}_{0}$. The analysis of conductance histograms shows that the most prominent and highest conductance peak found for all three molecular junctions is the one close to 2 $x 10^{-5} \mathrm{G}_{0}$. We attribute this value to the conductance through the Me-S terminated flat CCM backbone which equals $3.9 \times 10^{-5} \mathrm{G}_{0} \cdot{ }^{22}$

Initially, the close resemblance of the conductance values to the original CCM, without fullerene substituents, was unexpected considering the different conformation of the fullerene-based CCM skeleton (see Fig. 1 and optimized structures in Figs. S16). Specifically, in the CCM flat molecule with an enol structure, the two oxygen atoms are close due to the intramolecular hydrogen bonding (Fig. 1, top). Instead, the CCM$\mathrm{C}_{60}$ system presents a di-keto moiety (same for $\mathrm{CCM}-\mathrm{C}_{70}$ and $\mathrm{CCM}-\mathrm{C}_{60} \mathrm{P}$ ) displaying a $\mathrm{V}$-shape. The central carbon of this CCM skeleton is the apex and in opposite direction the two remaining halves of the molecule contain each a keto group (Figs. 1 and S16). It is also worth to note that the central carbon of the isolated CCM is involved in a delocalized $\pi$ bond while it has $\mathrm{sp}^{3}$ hybridization in the fullerocurcuminoid systems. Analysis of conductance traces (Fig. S12) corresponding to the fullerocurcuminoid systems are slightly shorter than those of the CCM molecule, in agreement with their modified V-shape structures. The additional lower-conductance peaks in CCM-C 70 and $\mathrm{CCM}-\mathrm{C}_{60}$ are longer and more slanted therefore they may be attributed to molecular junctions formed between one side of the Me-S backbone and the fullerene moieties. $\mathrm{CCM}-\mathrm{C}_{60} \mathrm{P}$ traces, on the other hand, show more straight plateaus (see Fig. S12). 
Additional insight from the conductance histograms can be obtained by performing a clustering analysis on the breaking-trace data sets (method II, see Table S2). For the three cases, the highest yield class (class 1) for each molecule corresponds to the conductance values close to those extracted using method I in the region of $1-4 \times 10^{-5}$ $\mathrm{G}_{0}$. It is worth noting that the highest yield class, considering the three systems, is class 1 of $\mathrm{CCM}-\mathrm{C}_{60} \mathrm{P}$, probably helped by the non-coordinative nature of bulky substituent leading to a higher number of measurements with the electrodes anchored to the two Me-S groups. In CCM-C 60 (Fig. S10c) the traces identified in the class 3 exhibit a broad distribution with conductance traces that decrease in value upon stretching (see Fig. S13 for example, breaking traces). Together with the small displacement involved and the relatively high conductance, this may indicate that the junction forms configurations mainly via the fullerene cage, most involving its sliding along the gold electrode. For $\mathrm{CCM}-\mathrm{C}_{70}$ similar high conductance features are found albeit at lower yield. Interestingly, the clustering does not show an additional low conductance feature as found in the filtering with method I. Further clustering of class one may be needed but for the analysis presented, however we have restricted the analysis to a maximum of four classes. The most important observation is the absence of clear lowor high conductance features for the $\mathrm{CCM}-\mathrm{C}_{60} \mathrm{P}$ derivative thereby reinforcing the hypothesis that the pathway through the fullerene moieties is obstructed.

DFT and non-equilibrium Green Function (NEGF) calculations (see Computational details section) were performed to understand why the highest conductance values of the CCM-fullerene family are similar to that of the CCM system. Table S3 shows the calculated conductance values for the $\mathrm{CCM}-\mathrm{C}_{70}, \mathrm{CCM}-\mathrm{C}_{60}$ and $\mathrm{CCM}$ systems. A wellknown drawback of the used PBE functional is that it provides small HOMO-LUMO energies, resulting in an overestimation of the conductance values, but giving reliable trends. The calculated conductance values for the most stable conformation in the junction through the two methylthio groups of the CCM for the $C C M-C_{70}, C C M-C_{60}$ 
and CCM systems show similar values, being slightly larger for the single CCM, in agreement with the experimental data. A V-shaped curcuminoid without the fullerene was also calculated, giving the same conductance value as the complete structure. This result corroborates the fact that the fullerene only plays a structural role distorting the planar CCM moiety while its contribution to the current is negligible. The perpendicular orientation of the methyl groups at the ends of the CCM species in all cases is the most stable structure when the molecules are coordinated to the electrode (see Figs. S15 and S16), while the coplanar orientation is the most favorable conformation for the isolated molecules.

Figure 3 displays the transmission curves and transmission eigenfunctions showing that the transport is mainly due to the LUMO channel. Transmission eigenfunctions with a defined transmission $(0 \leq \mathrm{T} \leq 1)$ which are obtained from the diagonalization of the transmission matrix. The total transmission is the sum of the eigenvalues of all transmission eigenfunctions. ${ }^{29}$ As expected, the calculated transmission curves using non-periodic models and hybrid B3LYP functional (see Fig. 3) show a larger HOMO-LUMO gap, however, the LUMO is still mainly responsible for the transport properties. The position of the LUMO orbital with respect to the Fermi level for all the systems is relatively similar (Fig. 3), and consequently, they lead to comparable conductance values (see Table S3 and details in SI). In the case of the CCM molecule, the analysis of the transmission eigenfunctions corresponding to the LUMO shows a delocalized $\pi$ distribution along the whole molecule. For the CCM-fullerene systems, delocalization is interrupted by the central $\mathrm{sp}^{3}$ carbon. The transmission eigenfunction appears delocalized along the whole backbone consistent with the conductance values found for such types of organic systems.

In addition, a detailed analysis of the calculated conductance values in Table S3 reveals two noticeable features: (i) There is an important influence of the relative orientation of the terminal methyl groups for the transport properties (see transmission curves calculated with the PBE functional for both conformers, coplanar and perpendicular 


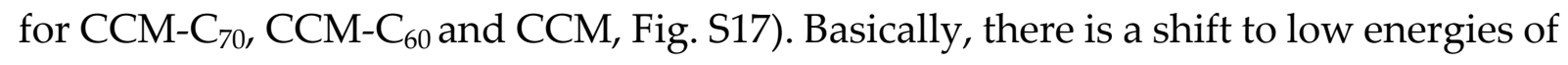
all the levels corresponding to the perpendicular conformer. Thus, the LUMO level is closer to the Fermi level for the perpendicular orientations leading to an increase of the conductance when compared with the coplanar case. In this regard, we have already shown that the orientation of the methyl groups of the anchoring Me-S ligands plays a key role in some systems. ${ }^{22}$ (ii) In the CCM system the central keto-enol unit favors $\pi$-delocalized systems with an odd number of atoms in the backbone showing $\pi$ delocalization over the whole molecule due to the central keto-enol $\left(\mathrm{C}_{3} \mathrm{O}_{2} \mathrm{H}\right)$ moiety.
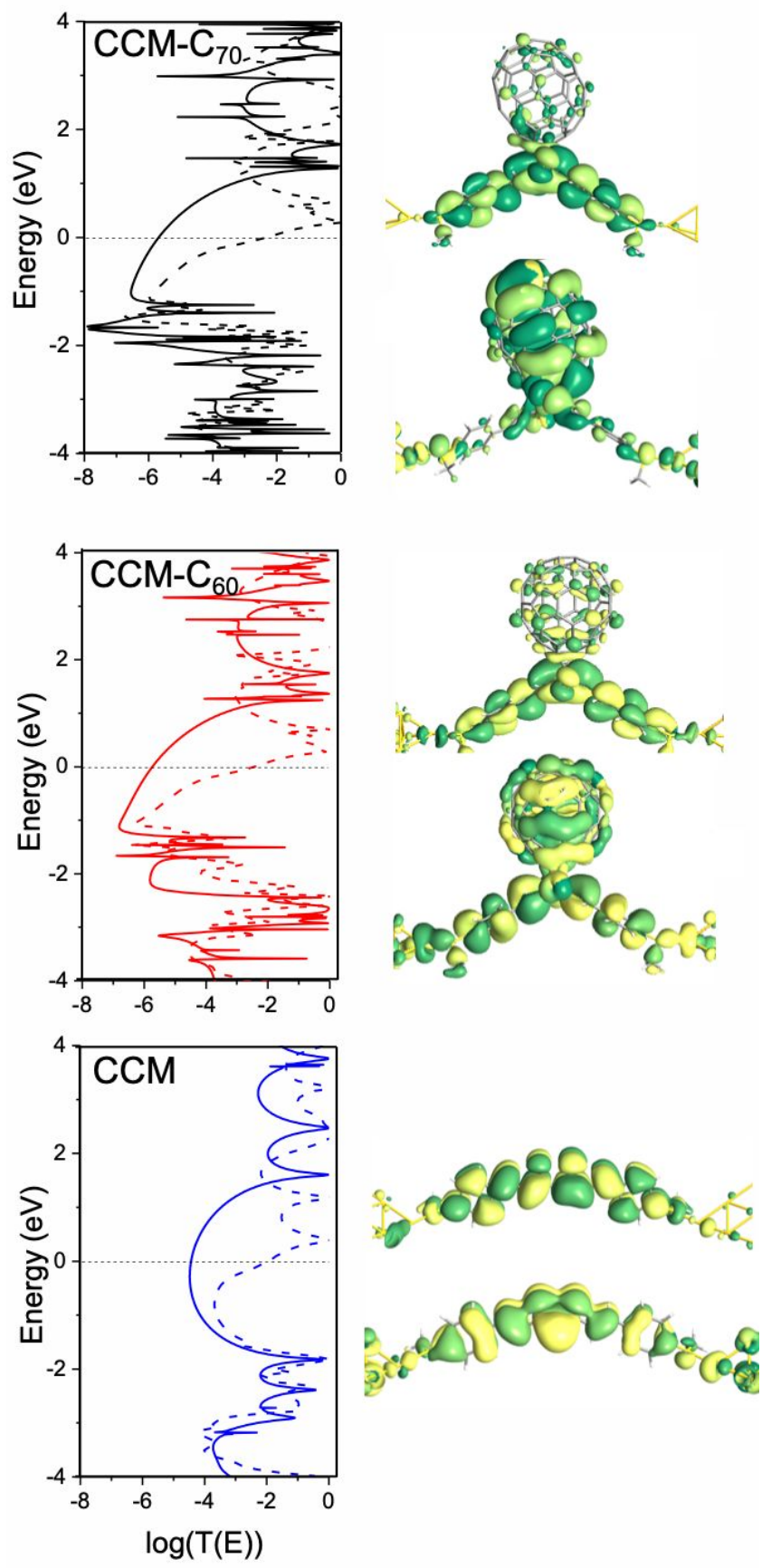
Figure 3. Transmission curves for $\mathrm{CCM}-\mathrm{C}_{70}, \mathrm{CCM}-\mathrm{C}_{60}$ and $\mathrm{CCM}$ derivatives calculated with the Artaios code using a non-periodic model with a wide band limit approach and B3LYP functional (continuous line) and with the ATK code using periodic models with the NEGF approach and PBE functional (dashed line). Transmission eigenfunctions of the PBE results were plotted for the frontier orbitals. The transmission eigenfunctions are obtained by diagonalizing the transmission matrix and the corresponding eigenvalues indicate the importance of each eigenstate in the transport. As it is a complex wavefunction, the color map indicates the phase of the function, represented from 0 to $2 \pi$ by dark green to yellow colors. The isovalue employed for the isosurface was 0.3 .

We have also analyzed the transmission pathways (Fig. 4) to gain insight into the transport mechanism considering the structural differences observed among the systems. Transmission pathways are calculated by the projection of the total transmission in pair contributions between atoms employing localized orbitals. This decomposition can be represented in real space by arrows indicating forward and backward current flow. ${ }^{30}$ We find that the transport mechanisms are different for the flat CCM in comparison to the nonplanar fullerocurcuminoid systems. In the CCM system, transport is directly through the neighboring carbon atoms but with some loss of current due to the two oxygens coordinated to the carbon backbone (destructive interference). However, in the fullerocurcuminoid systems transport through each keto unit is maintained as in the flat molecules, but with a direct tunneling pathway in the central region between the two external carbons of the $\mathrm{C}_{3} \mathrm{O}_{2}$ diketo unit (Fig. 4). This pathway, assisted by the shorter S...S distance due to the bending of the CCM system compensates for the nonflat structure of the CCM and results in a similar conductance value to that of the flat CCM system. In short, in the conjugated regions the electrons are travelling through the bonds, but in the non-conjugated parts of the molecule, i.e., the central carbon of the fullerocurcuminoid, the electrons are flowing around the bonds. ${ }^{31}$ 


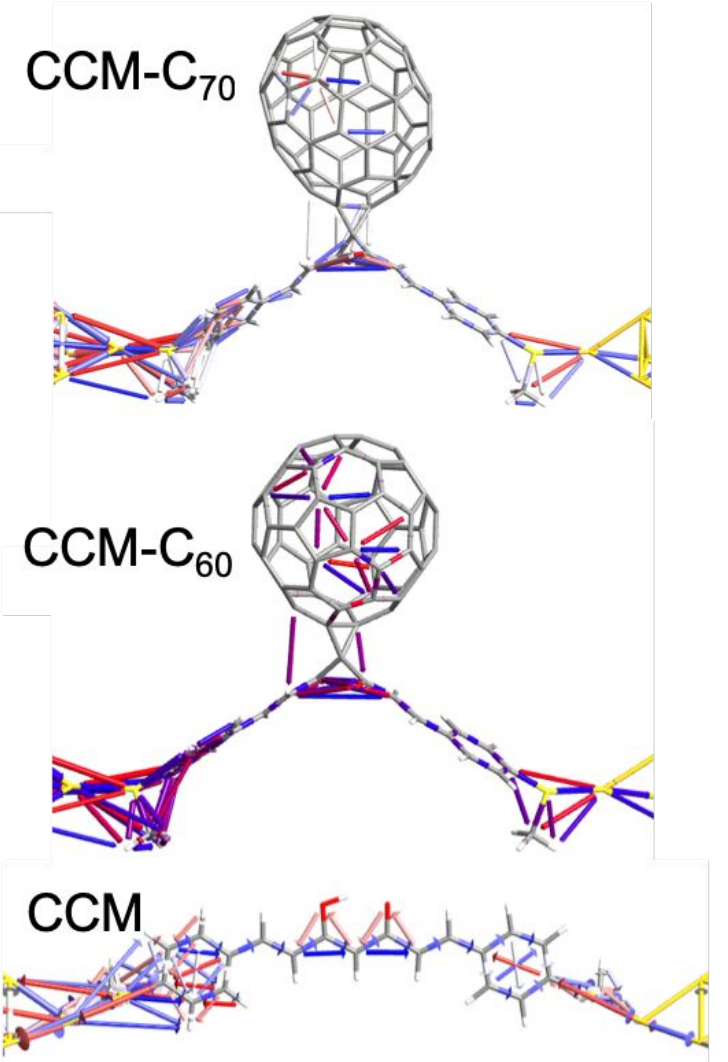

Figure 4. Transmission pathways for the systems based on $C C M-C_{70}, C C M-C_{60}$ and CCM. The color of the arrows (in CCM) indicates the angle of the direction of the transport, blue $\left(0^{\circ}\right.$, forwards $)$ and red $\left(180^{\circ}\right.$, backwards $)$, respectively and intermediate colors $\left(0-180^{\circ}\right)$. The width of the arrow is proportional to the magnitude of the transport. A threshold value of 0.0005 was employed.

Finally, the lowest conductance region has been explored by analyzing the sliding process of the gold electrode on the fullerene surface. For that, a total of three optimized structures taking into account the displacement of electrodes with respect to the molecule have been calculated (see Fig. S18). The decrease found in the calculated conductance values reproduces the experimental trend for $C \mathrm{CM}-\mathrm{C}_{70}$ and $\mathrm{CCM}-\mathrm{C}_{60}$; such values decrease while the electrode-molecule distance increases, with the fullerene and one of the branches of the V-shaped CCM attached to the gold electrodes (Fig. S18). Logically, the sliding process is not found in the $C C M-\mathrm{C}_{60} \mathrm{P}$ system as it is blocked by the malonate addents in agreement with the presence of horizontal plateaus in the experimental breaking traces plotted in Fig. S12. 
In conclusion, we demonstrated that fullerocurcuminoid molecular dyads can form multiple stable molecular junction configurations with well-defined conductance values and that equatorial diethyl malonate groups attached to the fullerene can block the transport pathway through it. A surprising finding is that both, $C_{70}$ and $C_{60}$, distort the planarity of the Me-S terminated CCM molecule drastically, however the conductance values through the dyads are very similar to the conductance of the planar CCM (around $10^{-5} \mathrm{G}_{0}$ ); our theoretical findings are in agreement with this observation. On one hand, CCM is planar and conjugated but there is a loss of current due to the two oxygens coordinated to the carbon backbone, which leads to destructive interference. On the other, in the CCM-fullerene systems there is a direct pathway between the two external carbons of the $\mathrm{C}_{3} \mathrm{O}_{2}$ diketo unit, assisted by the shorter $\mathrm{S} \cdots \mathrm{S}$ distance due to the bending of the CCM system, which compensates for the non-flat structure of the CCM. The stability of the MCBJ technique in combination with the filtering and clustering methods applied have been key in unravelling these features connecting structure with transport pathways.

\section{COMPUTATIONAL DETAILS}

Full consistent non-equilibrium Green function (NEGF) calculations ${ }^{32,33}$ were performed using the ATK program (2016.3 version). ${ }^{34}$ The PBE exchange-correlation functional ${ }^{35}$ was used together with numerical wavefunction of double- $\zeta$ quality with polarization for all the atoms except the gold atoms using a single- $\zeta$ quality with polarization. To calculate the conductance values assuming a linear regime, the experimental bias value used in the break-junction measurements was $0.10 \mathrm{~V}$. We have thus corroborated the linear regime between current and bias, and therefore the 
conductance can be easily obtained as one calculated value for a non-zero bias. The electrode structure employed in the calculations was a 2D $4 \times 4$ gold superlattice including a tip of 10 gold atoms on each electrode while a $4 \times 8$ superlattice was employed for the fullerene systems (see Fig. S16). A total number of 138 k-points was used to calculate the energy and wavefunction properties, while a $23 \times 152 \mathrm{D}$ grid was used for transport properties. The distances between the anchoring $S$ atoms to the gold atom of the tip was fixed to a value of $2.49 \AA$ for all the molecules. The molecules were optimized with Gaussian09 code $^{36}$ using B3LYP functional ${ }^{37}$ with a 6-311G* basis set. The position of the methyl of the anchoring group is coplanar for the geometry optimization of the isolated molecules but to reduce the repulsion with the gold electrodes a perpendicular disposition to the molecular plane was adopted. To confirm the role of the frontier orbitals in the transport (because GGA functionals show limitations to calculate HOMO-LUMO energies), we performed non-periodic calculations using hybrid density functionals. The transmission function was obtained in a post-processing routine through the Artaios ${ }^{38,39}$ program. Hamiltonian and overlap matrices needed for the calculation of the transmission function were obtained using ORCA 4.0.1 ${ }^{40}$ ) (B3LYP functional/def2-SVP basis set. ${ }^{41,42,43,}$ This transport code allows calculations with more accurate hybrid functionals improving the orbital energies but using non-periodic models with a rather simpler wide-band limit approximation. ${ }^{44}$ The fullerene-electrode contacts for the sliding process were optimized by using the tight-binding approach proposed by Grimme et al.. ${ }^{45}$

\author{
AUTHOR INFORMATION \\ *Email: diana.dulic@gmail.com , h.s.j.vanderzant@tudelft.nl
}

ACKNOWLEDGMENTS 
Financial support from the European Commission (COST Action MOLSPIN CA15128 and EU RISE (DAFNEOX) project SEP-210165479) is gratefully acknowledged. The work at University of Chile was supported by Fondecyt Regular Project 1181080 (D.D.), Fondecyt Regular Project 1161775 (M.S.), Fondecyt Regular Project 1170524 (D.A) , Fondequip EQM140055 and EQM180009 (D.D), Powered@NLHPC: This research was partially supported by the supercomputing infrastructure of the NLHPC (ECM-02). E.R. acknowledges MICIIN for grant PGC2018-093863-B-C21 and the Maria de Maeztu Excellence Grant MDM2017-0767, to the computer resources, technical expertise and assistance provided by the Barcelona Supercomputing Centre and CSUC and to the Generalitat de Catalunya for an ICREA Academia award and the grant 2017SGR1289. HvdZ acknowledges support from the Dutch Science foundation (NWO). N-A.-A. thanks MEC for grant MAT2016-77852-C2-1-R, to the Generalitat de Catalunya for the grant 2017SGR1277, and the Severo Ochoa Program for Centers of Excellence in R\&D (SEV-2015-0496). This project has received funding from the European Research Council (ERC) under the European Union's Horizon 2020 research and innovation program (grant agreement 724981). L.E thanks the US National Science Foundation (NSF) for generous support of this work under CHE-1801317 program. The Robert A. Welch Foundation is also gratefully acknowledged for an endowed chair to L. E. (Grant AH-0033). ABBREVIATIONS

$\mathrm{CCM}-\mathrm{C}_{70} /-\mathrm{C}_{60}$ fullerocurcuminoid molecular dyads; $\mathrm{CCM} / \mathrm{C}_{60} \mathrm{P}$, all-equatorial tetramalonate $\mathrm{C}_{60}$ fullerocurcuminoid molecular dyad; Me-S methylthio; DFT Density Functional Theory; NEGF

Non-Equilibrium Green Functions; MCBJ mechanically controllable break junction.

\section{REFERENCES}

(1) Grüter, L.; Cheng, F.; Heikkilä, T. T.; González, M. T.; Diederich, F.; Schönenberger, C.; Calame, M. Resonant Tunnelling Through a $\mathrm{C}_{60}$ Molecular Junction in a Liquid Environment. Nanotechnology 2005, 16, 2143-2148.

(2) Hirsch, A. The Era of Carbon Allotropes. Nat. Mater. 2010, 9, 868-871.

(3) Castro, E.; Martinez, Z. S.; Seong, C. S.; Cabrera-Espinoza, A.; Ruiz, M.; Hernandez, G., A; Valdez, F.; Llano, M.; Echegoyen, L. A. Impact of fullerene derivative isomeric purity on the performance of inverted planar perovskite solar cells. J. Med. Chem. 2016, 59, 10963-10973. 
(4) Castro, E.; Murillo, J.; Fernandez-Delgado, O.; Echegoyen, L. Organic interfacial materials for perovskite-based optoelectronic devices. J. Mater. Chem. C. 2018, 6, 2635-2651. (5) Li, C.-Z.; Matsuo, Y.; Nakamura, E. Octupole-Like Supramolecular Aggregates of Conical Iron Fullerene Complexes into a Three-Dimensional Liquid Crystalline Lattice. J. Am. Chem. Soc. 2010, 132, 15514-15515.

(6) Ballesteros, B.; de la Torre, G.; Shearer, A.; Hausmann, A.; Herranz, M. O.; Guldi, D. M.; Torres, T. Lanthanide(III) Bis(phthalocyaninato)-[60]Fullerene Dyads: Synthesis, Characterization, and Photophysical Properties. Chem. Eur. J. 2010, 16, 114-125.

(7) Halim, M.; Kennedy, R. D.; Khan, S. I.; Rubin, Y. Gold(I) Triphenylphosphine Complexes Incorporating Pentaarylfulleride Ligands. Inorg. Chem. 2010, 49, 39743976.

(8) Zhang, S.; Brown, T. L.; Du, Y.; Shapley, J. R. Metalation of Fullerene $\left(C_{60}\right)$ with Pentacarbonylrhenium Radicals. Reversible Formation of $\mathrm{C}_{60}\left\{\operatorname{Re}(\mathrm{CO})_{5}\right\}_{2}$. J. Am. Chem. Soc. 1993, 115, 6705-6709.

(9) Andersson, C.-H.; Nyholm, L.; Grennberg, H. Synthesis and Characterization of a Ferrocene-Linked Bis-Fullerene[60] Dumbbell. Dalton Trans. 2012, 41, 2374-2381.

(10) Joachim, C.; Gimzewski, J. K.; Schlittler, R. R.; Chavy, C. Electronic Transparence of a Single $\mathrm{C}_{60}$ Molecule. Phys. Rev. Lett. 1995, 74, 2102-2105.

(11) Böhler, T.; Edtbauer, A.; Scheer, E. Conductance of Individual $\mathrm{C}_{60}$ Molecules Measured with Controllable Gold Electrodes. Phys. Rev. B 2007, 76, 125432.

(12) Parks, J.; Champagne, A.; Hutchison, G.; Flores-Torres, S.; Abruna, H.; Ralph, D. Tuning the Kondo Effect with a Mechanically Controllable Break Junction. Phys. Rev. Lett. 2007, 99, 026601.

(13) Kiguchi, M.; Murakoshi, K. Conductance of Single $\mathrm{C}_{60}$ Molecule Bridging Metal Electrodes. J. Phys. Chem. C 2008, 112, 8140-8143.

(14) Bilan, S.; Zotti, L. A.; Pauly, F.; Cuevas, J. C. Theoretical Study of the Charge Transport Through $\mathrm{C}_{60}$-Based Single-Molecule Junctions. Phys. Rev. B 2012, 85, 205403. 
(15) Géranton, G.; Seiler, C.; Bagrets, A.; Venkataraman, L.; Evers, F. Transport Properties of Individual C 60 -Molecules. J. Chem. Phys. 2013, 139, 234701.

(16) Martin, C. A.; Ding, D.; Sørensen, J. K.; Bjørnholm, T.; van Ruitenbeek, J. M.; van der Zant, H. S. J. Fullerene-Based Anchoring Groups for Molecular Electronics. J. Am. Chem. Soc. 2008, 130, 13198-13199.

(17) Lörtscher, E.; Geskin, V.; Gotsmann, B.; Fock, J.; Sørensen, J.; Bjørnholm, T.; Cornil, J.; van der Zant, H.S.J.; Riel, H. Bonding and Electronic Transport Properties of Fullerene and Fullerene Derivatives in Break-Junction Geometries. Small 2013, 9, 209-214.

(18) Leary, E.; Gonzalez, M. T.; Van Der Pol, C.; Bryce, M. R.; Filippone, S.; Martín, N.; Rubio-Bollinger, G.; Agraït, N. Unambiguous One-Molecule Conductance Measurements Under Ambient Conditions. Nano Lett. 2011, 11, 2236-2241. (19) Moreno-García, P.; La Rosa, A.; Kolivoška, V.; Bermejo, D.; Hong, W.; Yoshida, K.; Baghernejad, M.; Filippone, S.; Broekmann, P.; Wandlowski, T.; Nazario, M. Charge Transport in $\mathrm{C}_{60}$-Based Dumbbell-Type Molecules: Mechanically Induced Switching Between Two Distinct Conductance States. J. Am. Chem. Soc. 2015, 137, 2318-2327.

(20) Morita, T.; Lindsay, S. Reduction-Induced Switching of Single-Molecule Conductance of Fullerene Derivatives. J. Phys. Chem. B 2008, 112, 10563-10572. (21) Stefani, D.; Gutiérrez-Cerón, C. A.; Aravena, D. ; Labra-Muñoz, J. ; Suarez, C. ; Liu, S. ; Soler, M.; Echegoyen, L. ; van der Zant, H. S. J; Dulić, D. Charge transport through a single molecule of trans-1-bis-diazofluorene [60] fullerene. Chemistry of Materials 2017, 29, 7305-7312.

(22) Olavarría-Contreras, I. ; Etcheverry-Berríos, A. ; Qian, W.; GutiérrezCerón, C.; Campos-Olguín, A.; Sañudo, E. C.; Dulić, D. ; Ruiz, E.; AliagaAlcalde, N.; Soler, M.; van der Zant, H. S. J. Electric-field induced bistability in single-molecule conductance measurements for boron coordinated curcuminoid compounds. Chem. Sci. 2018, 9, 6988-6996. 
(23) Etcheverry-Berríos, A.; Olavarría, I.; Perrin, M. L. ; Díaz-Torres, R.; Jullian, D. ; Ponce, I. ; Zagal, J. H. ; Pavez, J. ; Vásquez, S. O. ; van der Zant, H. S. J. ; Dulić, D.; Aliaga-Alcalde, N. ; Soler, M. Multiscale Approach to the Study of the Electronic Properties of Two Thiophene Curcuminoid Molecules. Chem., Eur. J. 2016, 22, 12808-12818.

(24) Castro, E.; Cerón, M. R.; Garcia, A. H.; Kim, Q.; Etcheverry-Berríos, A.; Morel, M. J.; Díaz-Torres, R.; Qian, W.; Martinez, Z.; Mendez, L.; Perez, F.; Santoyo, C. A.; Gimeno-Muñoz, R.; Esper, R.; Gutierrez, D. A.; Varela-Ramirez, A.; Aguilera, R. J.; Llano, M.; Soler, M.; Aliaga-Alcalde, N.; Echegoyen, L. A new family of fullerene derivatives: fullerene-curcumin conjugates for biological and photovoltaic applications. RSC Adv. 2018, 8, 41692-41698.

(25) Castro, E.; Azmani, K.; Hernández Garcia, A.; Aghabali, A.; Liu, S.; Metta-Magana, A.; Olmstead, M.; Rodriguez-Fortea, A.; Poblet, J.; Echegoyen, L., Unusual $\mathrm{C}_{2 \mathrm{~h}}$-symmetric trans-1-(bis-pyrrolidine)-tetra-malonate hexa-adducts of $\mathrm{C}_{60}$. The unexpected regio- and stereo-control mediated by malonate-pyrrolidine interaction. Chem. Eur. J. 2017, 23, 1593715944.

(26) Martin, C.A.; Ding, D.; van der Zant, H.S.J; van Ruitenbeek, J. M.; Lithographic mechanical break junctions for single-molecule measurements in vacuum: possibilities and limitations. New J. Phys. 2008, 10, 065008.

(27) Martin, C.A.; Smit, R.; Egmond, R.; van der Zant, H.S.J.; van Ruitenbeek, J.M.; A versatile low-temperature setup for the electrical characterization of single-molecule junctions

Rev. Sci. Instrum. 2011, 82, 053907.

(28) Cabosart, D.; El Abbassi, M.; Stefani, D.; Frisenda, R.; Calame, M.; van der Zant, H.S.J.; Perrin, M.L.; A reference-free clustering method for the analysis of molecular break-junction measurements. Appl. Phys. Lett. 2019, 114, 143102.

(29) Paulsson, M.; Brandbyge, M. Transmission Eigenchannels from Nonequilibrium Green's Functions. Phys. Rev. B 2007, 76, 115117.

(30) Solomon, G. C.; Herrmann, C.; Hansen, T.; Mujica, V.; Ratner, M. A. Exploring Local Currents in Molecular Junctions. Nat. Chem. 2010, 2, 223-228 
(31) Jensen, A.; Garner, M. H.; Solomon, G. C. When Current Does Not Follow Bonds: Current Density in Saturated Molecules. J. Phys. Chem. C. 2019, 123, 12041-12051

(32) Cuevas JC, Scheer E (2010) Molecular Electronics: An Introduction to Theory and Experiment World Scientific Series in Nanotechnology and Nanoscience. World Scientific Publishing Company, Singapore.

(33) Brandbyge, M.; Mozos, J. L.; Ordejon, P.; Taylor, J.; Stokbro, K., Density-functional method for nonequilibrium electron transport. Physical Review B 2002, 65, 165401.

(34) Atomistix ToolKit ATK, 2016.3; QuantumWise A/S: 2016.

(35) Perdew, J. P.; Burke, K.; Ernzerhof, M., Generalized gradient approximation made simple. Phys. Rev. Lett. 1996, 77, 3865-3868.

(36) Frisch, M. J.; Trucks, G. W.; Schlegel, H. B.; Scuseria, G. E.; Robb, M. A.; Cheeseman, J. R.; Scalmani, G.; Barone, V.; Mennucci, B.; Petersson, G. A.; Nakatsuji, H.; Caricato, M.; Li, X.; Hratchian, H. P.; Izmaylov, A. F.; Bloino, J.; Zheng, G.; Sonnenberg, J. L.; Hada, M.; Ehara, M.; Toyota, K.; Fukuda, R.; Hasegawa, J.; Ishida, M.; Nakajima, T.; Honda, Y.; Kitao, O.; Nakai, H.; Vreven, T.; Montgomery Jr., J. A.; Peralta, J. E.; Ogliaro, F.; Bearpark, M. J.; Heyd, J.; Brothers, E. N.; Kudin, K. N.; Staroverov, V. N.; Kobayashi, R.; Normand, J.; Raghavachari, K.; Rendell, A. P.; Burant, J. C.; Iyengar, S. S.; Tomasi, J.; Cossi, M.; Rega, N.; Millam, N. J.; Klene, M.; Knox, J. E.; Cross, J. B.; Bakken, V.; Adamo, C.; Jaramillo, J.; Gomperts, R.; Stratmann, R. E.; Yazyev, O.; Austin, A. J.; Cammi, R.; Pomelli, C.; Ochterski, J. W.; Martin, R. L.; Morokuma, K.; Zakrzewski, V. G.; Voth, G. A.; Salvador, P.; Dannenberg, J. J.; Dapprich, S.; Daniels, A. D.; Farkas, Ö.; Foresman, J. B.; Ortiz, J. V.; Cioslowski, J.; Fox, D. J.; Gaussian, Inc.: Wallingford, CT, USA, 2009.

(37) Becke, A.D. Density-functional thermochemistry .3. The role of the exact exchange. J. Chem. Phys. 1993, 98, 5648-5652.

(38) Deffner M.; Groß, L; Steenbock, T.; Voigt, B. A.; Solomon, G. C.; Herrmann, C. Artaios - a code for postprocessing quantum chemical electronic structure calculations, available from https://www.chemie.uni-hamburg.de/ac/herrmann/software/index.html (2008-2017).

(39) Herrmann, C. ; Solomon, G.C.; Subotnik, J.E. ; Mujica, V. ; Ratner, M.A. Ghost transmission: How large basis sets can make electron transport calculations worse. J. Chem. Phys. 2010, 132, 024103. 
(40) Neese, F. Software update: the ORCA program system, version 4.0 Wiley Insterdiscip. Rev. Comput. Mol. Sci. 2017, 8, 1327.

(41) Schäfer, A.; Horn, H.; Ahlrichs, R. Fully Optimized Contracted Gaussian Basis Setsfor Atoms Li to Kr. J. Chem. Phys. 1992, 97, 2571-2577.

(42) Schwerdtfeger, P.; Dolg, M.; Schwarz, W. E.; Bowmaker, G. A.; Boyd, P. D. Relativistic Effects in Gold Chemistry. I. Diatomic Gold Compounds. J. Chem. Phys. $1989,91,1762-1774$.

(43) Weigend, F.; Ahlrichs, R. Balanced Basis Sets of Split Valence, Triple Zeta Valence and Quadruple Zeta Valence Quality for H to Rn: Design and Assessment of Accuracy. Phys. Chem. Chem. Phys. 2005, 7, 3297-3305.

(44) Martín-Rodríguez, A.; Aravena, D.; Ruiz, E. DFT approaches to transport calculations in magnetic single-molecule devices. Theor. Chem, Acc. 2016, 135,192.

(45) Grimme, S.; Bannwarth, C.; Shushkov, P. A Robust and Accurate Tight-Binding Quantum Chemical Method for Structures, Vibrational Frequencies and Noncovalent Interactions of Large Molecular Systems Parametrized for All spd-Block Elements (Z=1-86). J. Chem. Theor. Comput. 2017, 12, 1989-2009. 